Alfarama Journal of Basic \& Applied Sciences

Faculty of Science Port Said University https://ajbas.journals.ekb.eg

ajbas@sci.psu.edu.eg

http://sci.psu.edu.eg/en/
January 2022, Volume 3 Issue I

Accepted: 08-11-2021
DOI: $10.21608 / A J B A S .2021 .99552 .1071$

DOI: $10.21608 / A B A S .2021 .99552 .1071$

\section{Submitted: 04-10-2021}

Accepted:

Pages: 131-137

\title{
Fractional order COVID 19 pandemic model in Morocco: Dynamical analysis and Numerical simulation
}

\author{
A.M.A.El-Sayed ${ }^{1}$, I. M. Hanafy ${ }^{3}$, A. A. M. Arafa ${ }^{2,3}$ and M.I.Gouda ${ }^{3, *}$ \\ ${ }^{1}$ Department of Mathematics, Faculty of Science, Alexandria university, Alexandria, Egypt \\ ${ }^{2}$ Department of Mathematics, College of Sciences and Arts, Methnab, Qassim University, P.O. Box 931, \\ Buridah, 51931, Methnab, Kingdom of Saudi Arabia \\ ${ }^{3}$ Department of Mathematics and Computer Science, Faculty of Science, Port Said University, Port Said, \\ Egypt \\ "Corresponding author: gouda2016@gmail.com
}

\begin{abstract}
In this paper, we introduce fractional order COVID-19 model in Morocco. We analyse the presented model by calculating reproductive ratio $R_{0}$, finding free disease equilibrium point and studying local and global stability of free disease equilibrium point $E_{0}$ to show the advantages of fractional order model, we simulate model by using (PECE) method for fractional order and compare our results to clinical data which obtained from morocco cases from 18 march 2020 to 5 April 2020 . From presented figures, we can easily see that data from fractional order is better than data obtained by integer order model according to the comparison with clinical data
\end{abstract}

Keywords

COVID-19 fractional order model, Fractional Adams-type predictor-corrector method, boundedness of solution, local and global stability analysis, Numerical results.

\section{INTRODUCTION}

Recently, the World Health Organization (WHO) declared that COVID-19 has become a pandemic [1]. The epidemic is described as occurring in a wide geographical area and affecting an unusually high proportion of the population. [2]. On December 31, 2019, groups of cases were reported with pneumonia of a mysterious cause, in Wuhan, Hubei Province, China. In January 2020, tests obtained from cases and examination of the genetic characteristics of the infection showed that it was a new highly infectious virus [3-4]. This new virus was named Coronavirus Disease 2019 (COVID-19) by the World Health Organization in February 2020[3]. Coronaviruses are a group of infections that cause diseases, for example, respiratory diseases or infections of the digestive system. Covids got their name from the way they look under a magnifying tool. The infection consists of a center of genetic material surrounded by (Middle East Respiratory Syndrome (MERS-CoV), Severe Acute Respiratory Syndrome (SARS-CoV)) [9]. (NCOV) is another strain that has not been distinguished in people beforehand. When researchers decide precisely what Covid is, they give it a name (as on account of COVID-19, the an envelope with protein mutations. This gives her the presence of the crown. Corona viruses are zoonotic [6], which means that the infection is transmitted between creatures and humans. It has been found that the Corona virus that causes respiratory syndrome in the Middle East has 
been transmitted from Arab camels to humans, and the SARS virus from civet strains to humans [7]. The source of SARS-CoV-2 (COVID-19) has not yet been resolved, however, checks are ongoing to identify the animal source of the outbreak [8]. In the course of recent months, a few works of numerical displaying have been distributed taking into account the uncovered and asymptomatic classes [9-13].The fractional derivatives have several different kinds of definitions, among which the Riemann-Liouville fractional derivative and the Caputo fractional derivative are two of the most important ones in applications [12]. The Risezctional derivative is a linear representation of the left Riemann-Liouville fractional derivative and right Riemann-Liouville fractional derivative. A close relationship exists between the Riemann-Liouville fractional derivative and the Caputo fractional derivative. The Riemann-Liouville fractional derivative can be converted to the Caputo fractional derivative under some regularity assumptions of the function $[12,13]$. In fractional partial differential equations, the time-fractional derivatives are commonly defined using the Caputo fractional derivatives. The main reason lies in that the Riemann-Liouville approach needs initial conditions containing the limit values of Riemann-Liouville fractional derivative at the origin of time $t=0$, whose physical meanings are not very clear. However, in cases with the time-fractional Caputo derivative, the initial conditions take the same form as that for integer-order differential equations, namely, the initial values of integer-order derivatives of functions at the origin time. In recent years, there are many biological models that have been modelled using fractional orders because they are concerned with memory [14-19]. During recent months, many papers studying COVID-19 disease with fractional order have been published [9,18-23]. In [11] the authors consider broader categories together to consider the effectiveness of overall conclusion and assess our control system that will apply to all individuals regardless of their disclosure status or not. The first category is the aware people who think about the infection and the cross-country lockdown, called the mostly controlled people $(S p)$, since this individual could not regard the public isolate for some explanation also, could be considered as law violators. The subsequent class is individuals who regard the public isolate by remaining at home from the beginning of the conclusion. The individuals are known as a completely controlled individual $S_{c}$. Other categories are infected individuals $(t)$, removed individuals $(t)$ and dead people $N_{d}(t)$. In this paper, we introduce fractional COVID-19 model as following equations:

$$
\begin{aligned}
& D^{\alpha} S=-\frac{\beta S I}{N}-\frac{\lambda S}{(1-q S)}+k R-v S, \\
& D^{\alpha} S_{p}=-\frac{\mu \beta S_{p} I}{N}+\frac{\lambda_{1} S}{(1-q S)}-v S_{p}, \\
& D^{\alpha} S_{c}=\frac{\lambda_{2} S}{(1-q S)}+v\left(S_{p}+S\right), \\
& D^{\alpha} I=\frac{\beta}{N}\left(S+\mu S_{p}\right) I-(\varepsilon+\tau+\rho) I, \\
& D^{\alpha} R=(\varepsilon+\tau) I-k R, \\
& D^{\alpha} N_{d}=\rho I .
\end{aligned}
$$

where $N(t)=S+S_{p}+S_{c}+I+R$ with initial values of $S(0), S_{p}(0), S_{c}(0), I(0), R(0)$ and $N_{d}(0) \geq 0, \mathrm{~S}$ is susceptible individuals, $S_{p}$ is partially controlled susceptible individuals, $S_{c}$ totally controlled susceptible individuals, $\mathrm{I}$ is infected individuals, $\mathrm{R}$ is removed individuals according to recovery or death without COVID-19, $N_{d}$ is dead people because of COVID-19 infection $\lambda_{1}$ is transition rate from susceptible to partially controlled category, $\lambda_{2}$ is transition rate from susceptible to totally controlled category, $\lambda=\lambda_{1}+$ $\lambda_{2}, 0<\mu \leq 1$ is infection rate of partially controlled individuals, $\beta$ is the spreading COVID-19 rate, $\rho$ is death rate by COVID-19, $\tau$ is the curing rate, $\varepsilon$ is healing rate, $\kappa$ is immunodeficiency rate, $\alpha$ is The inhibition effect due to resource limitation to awareness controls population and $v$ is the effect of curfew. All previous parameters are calculated and found in [11]. The fractional order $0<\alpha \leq 1$, and $D^{\alpha}$ is called Caputo fractional derivative sense [12-17], which defined, as: 
$D^{\alpha} f(x)=\frac{1}{\Gamma(n-\alpha)} \int_{0}^{x}(x-t)^{n-\alpha-1} f^{(n)}(t) d t$,

for $n-1<\alpha \leq n, n \in N, x>0$

\section{BOUNDENESS OF SOLUTION}

Defination 2.1. Let $\boldsymbol{f}(\boldsymbol{x})$ denote a function which vanishes for negative values of $\boldsymbol{x}$. Its Laplace's transform $\boldsymbol{L}_{\boldsymbol{\alpha}}\{\boldsymbol{f}(\boldsymbol{x})\}$ of order $\boldsymbol{\alpha}$ (or its $\boldsymbol{\alpha}$-th fractional Laplace's transform) is defined by the following expression, when it is finite:

$$
L_{\alpha}\left\{f(x):=: F_{\alpha}(s)=\int_{0}^{\infty} E_{q}\left(-s^{\alpha} x^{\alpha}\right) f(x)(d x)^{\alpha}\right.
$$

Theorem 2.1. suppose that $S(t), S_{p}(t), S_{c}(t), I(t), R(t)$ and $N_{d}(t)$ are non-negative for all $t \geq 0$ then the solution is bounded.

Proof

Suppose that $N_{1}(t)=S(t)+S_{p}(t)+S_{c}(t)+I(t)+R(t)+N_{d}(t), D^{\alpha} N_{1}=0$

We have:

$$
\begin{gathered}
N_{1}(t)=N_{1}(0), N(t) \leq N_{1}(t)=N_{1}(0) . \\
D^{\alpha} N_{d}(t)=\rho I \leq(1+\rho) N-N_{d} \leq(1+\rho) N_{1}(0)-N_{d}
\end{gathered}
$$

By using Laplace transformation, we get:

$$
N_{d}(t) \leq\left(N_{d}(0)-(1+\rho) N_{1}(0)\right) E_{q}\left(-t^{q}\right)+(1+\rho) N_{1}(0)
$$

Where $E_{q}\left(-t^{q}\right)$ is the Mittag-Leffer function of parameter $\alpha$. Then we have $I(t) \leq 0 \forall t>0$. If $N_{d}(0)<$ $(1+\rho) N_{1}(0)$ then $N_{d}(t) \leq(1+\rho) N_{1}(0)$. In case $N_{d}(0)>(1+\rho) N_{1}(0), \lim _{t \rightarrow \infty} E_{q}\left(-t^{\alpha}\right)=0$ then $N_{d}(t) \leq(1+\rho) N_{1}(0)$.

Proof complete.

\section{FREE DISEASE EQUILIBRIUM POINT AND REPRODUCTIVE RATIO}

In case free disease equilibrium point, no disease in population so that $S+S_{p}+S_{c}=N$ and $=R=N_{d}=0$ . The free disease equilibrium point is:

$$
E_{0}=\left(S_{0}, S_{p_{0}}, S_{c_{0}}, I_{0}, R_{0}, N_{d_{0}}\right)=\left(\frac{1}{\alpha}\left(\frac{\lambda}{v}+1\right), \frac{\lambda_{1}}{q \lambda}\left(\frac{\lambda}{v}+1\right), S_{c_{0}}, 0,0,0\right) .
$$

We can obtain $R_{0}$ as:

$$
R_{0}=\frac{\beta\left(S_{0}+\mu S_{p_{0}}\right)}{N_{0}(\varepsilon+\tau+\rho)}<\frac{\beta N_{1}(0)}{N_{0}(\varepsilon+\tau+\rho)}
$$

Where $S_{0}=\frac{1}{q}\left(\frac{\lambda}{v}+1\right), S_{p_{0}}=\frac{\lambda_{1}}{q \lambda}\left(\frac{\lambda}{v}+1\right)$ and $N_{0}=S_{0}+S_{p_{0}}+S_{c_{0}}$

\section{LOCAL STABILITY OF FREE DISEASE EQUILIBRIUM POINT}

Theorem 4.1: The equilibrium point $E_{0}$ is locally asymptotically stable if $R_{0}<1$.

Proof: 
The Jacobian matrix of system (1.1) at equilibrium point $E_{0}$ :

$$
J\left(E_{0}\right)=\left(\begin{array}{cccc}
\frac{-\lambda-v}{\left(1-q S_{0}\right)} & 0 & -\frac{\beta S_{0}}{N_{0}} & k \\
\frac{\lambda_{1}}{\left(1-q S_{0}\right)} & -v & -\frac{\mu \beta S_{p_{0}}}{N_{0}} & 0 \\
0 & 0 & \left.\frac{\beta}{N_{0}}\left(S+\mu S_{p_{0}}\right)-(\varepsilon+\tau+\rho)\right) & 0 \\
\frac{-\lambda-v}{\left(1-q S_{0}\right)} & 0 & -\frac{\beta S_{0}}{N_{0}} & k
\end{array}\right)
$$

The eigenvalues corresponding to the equilibrium $E_{0}$ are:

$$
\rho_{1}=-\frac{\lambda+v}{\left(1-q S_{0}\right)}, \rho_{2}=-v, \rho_{3}=-k, \rho_{4}=\frac{\beta}{N_{0}}\left(S_{0}+\mu S_{p_{0}}\right)-(\varepsilon+\tau+\rho)=(\varepsilon+\tau+\rho)\left(R_{0}-1\right)
$$

So that $\rho_{4}$ negative, if and only if $R_{0}<1$ and $\rho_{1}, \rho_{3}$ are negative where all parameters are positive. So $E_{0}$ is locally asymptotic stable if and only if $R_{0}<1$.

\section{GLOBAL STABILITY OF FREE DISEASE EQUILIBRIUM POINT}

Theorem 5.1. If $R_{0}<1$, then the disease-free equilibrium $E_{0}$ is globally asymptotically stable.

Proof.

For system (1.1), we get the following Lyapunov function:

$$
\begin{gathered}
V_{0}(t)=I(t) \\
D^{\alpha} V_{0}(t)=\frac{\beta}{N}\left(S+\mu S_{p}\right) I-(\varepsilon+\tau+\rho) I \\
D^{\alpha} V_{0}(t)<(\varepsilon+\tau+\rho) I\left(\frac{\beta N_{1}(0)}{N_{0}(\varepsilon+\tau+\rho)}-1\right)<0, \text { if } \frac{\beta N_{1}(0)}{N_{0}(\varepsilon+\tau+\rho)}<1 \text { then } R_{0}<1
\end{gathered}
$$

We have $V_{0}(t)>0, D^{\alpha} V_{0}(t)=0$ at $E_{0}$ and $D^{\alpha} V_{0}<0$, then $E_{0}$ is globally asymptotically stable according to [14] when $R_{0}<1$, which implies the disease will be disappear regardless the initial infected individuals.

\section{NUMERICAL SIMULATION}

We use Adams-type predictor-corrector method which explained in details in [14] to simulate system (1.1) and compare our results with clinical data. The initial conditions and parameter are represented in the following table: 
Table 1:

\begin{tabular}{|c|c|}
\hline parameter & Estimated value \\
\hline$\beta$ & 0.3090 \\
\hline$\mu$ & 0.790453 \\
\hline$\lambda_{1}$ & 0.198352 \\
\hline$\lambda_{2}$ & 0.060999 \\
\hline$\rho$ & 0.01065 \\
\hline$\tau$ & 0.00853 \\
\hline$\varepsilon$ & 0.001127 \\
\hline $\boldsymbol{\kappa}$ & $3.074789 \times 10^{-14}$ \\
\hline$q$ & $8.7726 \times 10^{-9}$ \\
\hline $\boldsymbol{v}$ & 0.037235 \\
\hline$S(\mathbf{0})$ & 34500000 \\
\hline $\boldsymbol{S}_{p}(\mathbf{0})$ & 0 \\
\hline$S_{c}(\mathbf{0})$ & 0 \\
\hline$I(0)$ & 63 \\
\hline $\boldsymbol{R}(\mathbf{0})$ & 2 \\
\hline$N_{d}(\mathbf{0})$ & 2 \\
\hline
\end{tabular}

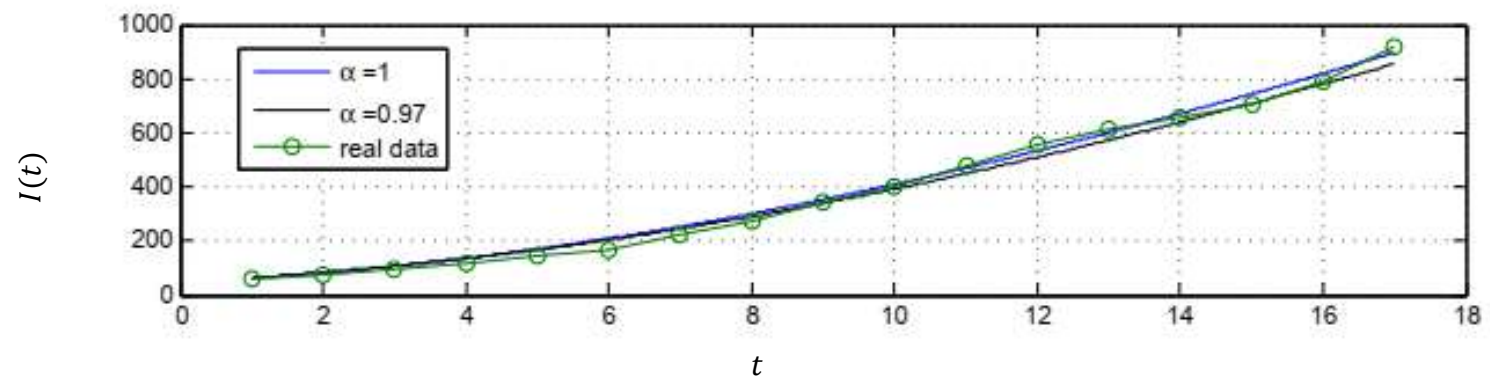

Figure 1. The relation between infected individuals $\mathrm{I}(\mathrm{t})$ and time $\mathrm{t}$ with integer order (blue line), fractional order (black line) and real data (green line). Data obtained from 18 March 2020 to 5 April 2020.

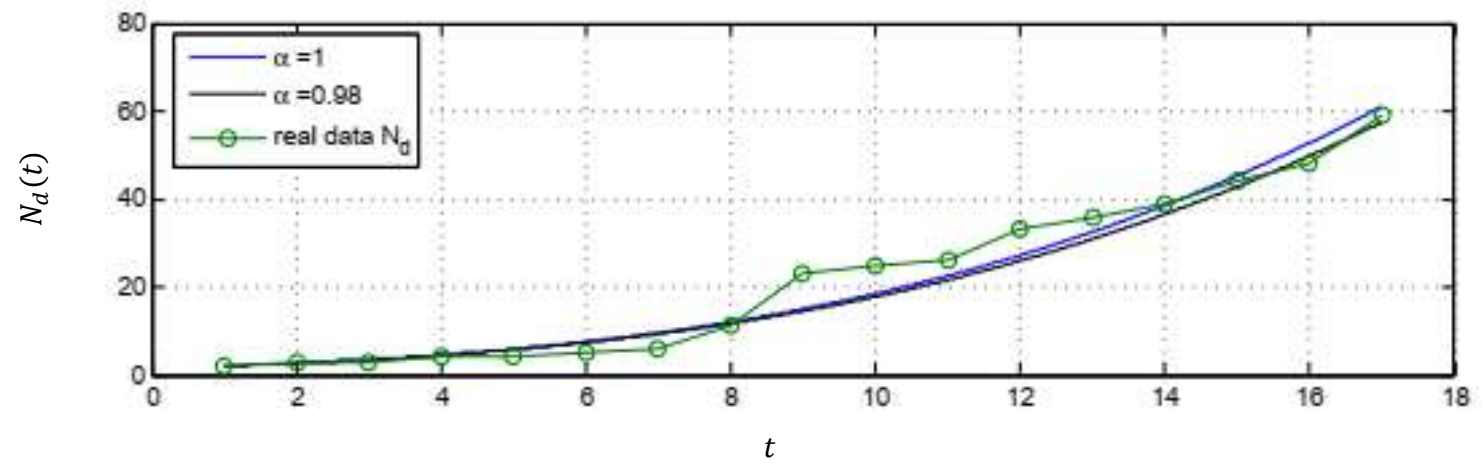

Figure 2. The relation between dead individuals $N_{d}(t)$ and time $t$ with integer order (blue line), fractional order (black line) and real data (green line). Data obtained from 18 March 2020 to 5 April 2020. 


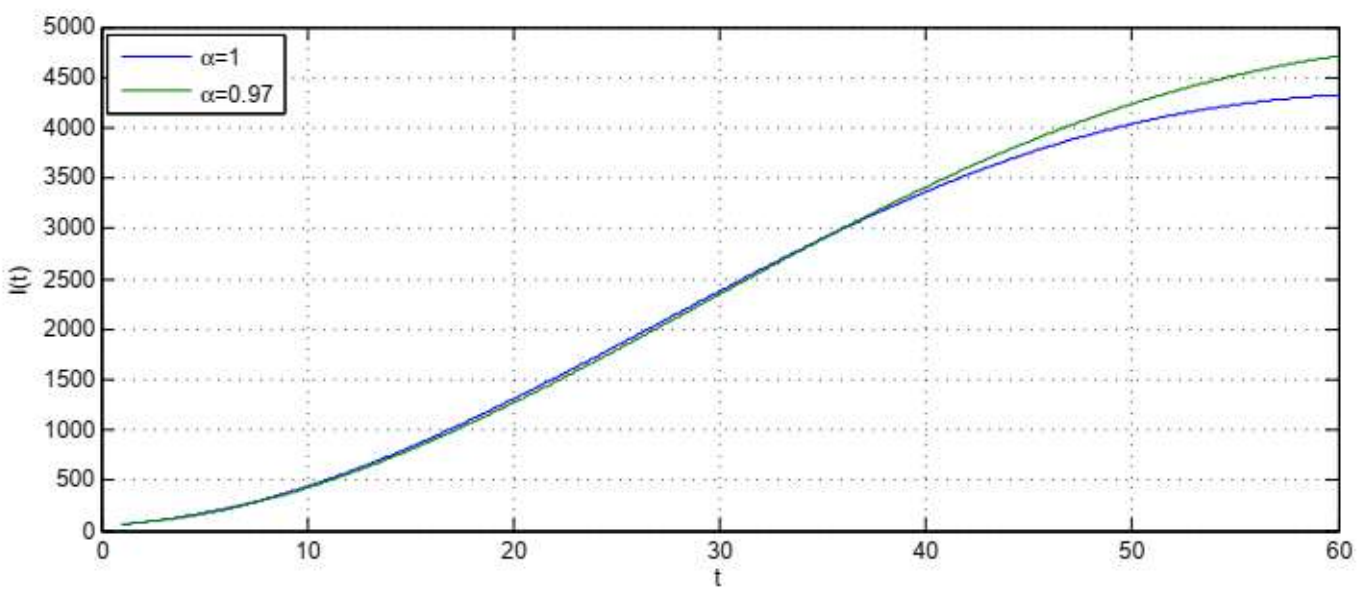

Figure 3. The relation between infected individuals $I(t)$ and time $t$ with integer order (blue line), fractional order (green line) predicted data from 18 march 2020 to 18 may 2020.

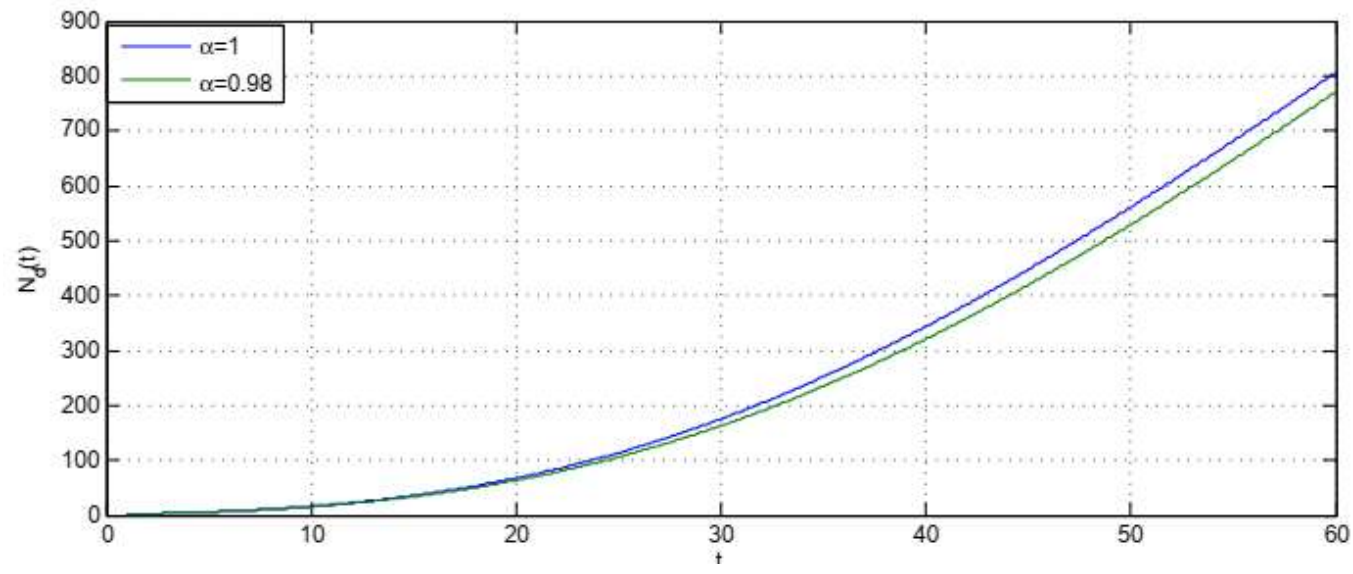

Figure 4. The relation between dead individuals $N_{d}(t)$ and time $\mathrm{t}$ with integer order (blue line), fractional order (green line) predicted data from 18 march 2020 to 18 may 2020.

\section{CONCULSION}

A fractional-order model for COVID-19 which supported with clinical data of morocco is introduced in this paper. Also, the basic reproduction number $R_{0}$, positively solution, boundedness of solution and global stability of the disease-free equilibrium have been studied. The numerical results showed the impact of fractional order in our results. In figure 1 and 2 show that data which obtained from fractional order model is slightly better than data from integer by comparison with clinical data. Figure 3 and 4 show the prediction of the number of infected individuals and dead people by COVID-19 infection. Fractional order model has large peak than integer order which agree with the behaviour of spreading of COVID-19. non-local fractional order is big advantage in disease modelling, we can choose more appropriate fractional order according to comparison with clinical data. Finally, we want to mention that we can't study endemic equilibrium point by local and global stability because we study the spread of disease with unstable of number of infected individuals .

\section{REFERENCES}

[1] Zakary, O. , Rachik, M., Elmouki, I.,(2017), Infectious Disease Modelling, vol. 2, no. 3, pp. 304322. https://doi.org/10.1016/j.idm.2017.06.003.

[2] Zakary, O., Rachik, M. , Elmouki, I.,(2017),Mathematical Methods in the Applied Sciences, vol. 40, no. 4, pp. 1265-1279. https://doi.org/10.1002/mma.4048. 
[3] Zakary, O., Larrache, A., Rachik, M.,Elmouki, I.,(2016), Advances in Difference Equations, vol. 2016, no. 1, 169-174. https://doi.org/10.1186/s13662-016-0900-9 .

[4] Cheng, V. C. C., To, K. K. W., Tse, H., Hung, I. F. N., Yuen, K. Y.,( 2012),Clinical Microbiology Reviews, vol.25, no. 2, pp. 223-263.https://doi.org/ 10.1128/CMR.05012-11

[5] Cheng, V. C. C., Tai, J. W. M. , . Lee W. M ,et al., (2015),Infection Control \& Hospital Epidemiology, vol. 36, no. 1, pp. 87-92. https://doi.org/10.1155/2020/9813926

[6] Khajji, B., Kada, D., Balatif, O. , Rachik, M.,( 2020),Journal of Applied Mathematics and Computing, vol. 64, no. 1-2, pp. 255-281.https://doi.org/10.1007/s12190-020-01354-3.

[7] Yu P, Zhu J, Zhang Z, Han Y. A.,( 2020) , J Infect Dis. Vol. 11,no(11):1757-1761. doi: 10.1093/infdis/jiaa077.

[8] Tuite, A. R., Fisman, D. N. \& Greer, A. L., (2020), CMAJ .Vol.192,no(11),E497-E505. https://doi.org/10.1503/cmaj.200476.

[9] Shaikh, A. S., Shaikh, I. N.,Nisar, K. S., (2020), Adv Differ Equ, vol. 2020, no. 1, 115-117. https://doi.org/10.1186/s13662-020-02834-3.

[10] Kouidere, A., Khajji, B., El Bhih, A., Balatif, O. , Rachik, M., (2020),Communications in Mathematical Biology and Neuroscience, vol. 2020, no.2020,1120-1130. https://doi.org/10.1155/2020/1943410.

[11] Zakary , O., Bidah , S., Rachik , M., Ferjouchia, H.,( 2020),Journal ofApplied Mathematics, vol.2020,no.2020,117-125. https://doi.org/10.1155/2020/9813926

[12] Arafa, A. A. M., Rida, S. Z., (2012), Appl. Math. Model, vol.36,no.10, 4789-4796. http://doi.org/10.1016/j.apm.2011.12.014

[13] Arafa, A.A.M, Khalil, M., Sayed, A., (2019) ,Complexity, Vol.2019,No.2019,13 pages. https://doi.org/10.1155/2019/4291017

[14] El-Saka, H.A.A, Arafa, A.A.M, Gouda, M.I, (2019), Adv Differ Equ ,Vol.2019,No.1 , 144-156. https://doi.org/10.1186/s13662-019-2079-3

[15] El-Sayed, A.M. A., Arafa, A A. M., Khalil, M.,Sayed,Amaal (2017),Progr. Fract. Differ.Appl.Vol. 3, No. 4, 281-287 .https://doi.org/10.18576/pfda/030404

[16] El-Sayed, A.M. A., Arafa , A. A. M., Khalil, M. Hassan, A., (2016),Progr. Fract. Differ. Appl.,Vol. 2, No. 2, 105-113. http://dx.doi.org/10.18576/pfda/020203

[17] Arafa, A.A.M., Rida, S.Z., Khalil, M.,(2014), International Journal ofBiomathematics, Vol.7,No.3 ,11 pages.https://doi.org/10.1155/2017/8372140

[18] Rajagopal,K., Hasanzadeh, N., Parastesh, F. et al., (2020), Nonlinear Dyn ,Vol.101, 711-718. https://doi.org/10.1007/s11071-020-05757-6

[19] I.Ahmed, I.A. Baba, A.Yusuf, et al., (2020),Adv Differ Equ, Vol.394, https://doi.org/10.1186/s13662-020-02853-0

[20] Higazy, M. , (2020), Chaos, Solitons \& Fractals, Vol.138, 7-11.https://doi: 10.1016/j.chaos.2020.110007

[21] Ali, Z., Rabiei, F., Shah, K. ,Kodadadi, T.,(2021),Alexandia Engineering Journal,Vol. 60, No.1,477-489. https://doi.org/10.1016/j.aej.2020.09.020

[22] Bahloul, M. A. , Chahid, A. Laleg-Kirati \& T.-M. ,(2020),IEEE Open Journal of Engineering inMedicine and Biology, Vol.1, No.1,249-256. https://doi.org/10.1011/j.aej.2020.08.034

[23] Higazy, M., Alyami, M.,(2020),Alexandria Engineering Journal, Vol.59,No.6,4719-4736. https://doi.org/10.1016/j.aej.2020.08.034 